\title{
LA DISTRIBUCIÓN DEL IMPUESTO A LA RENTA ENTRE PERÍODOS
}

\author{
DISTRIBUTION OF INCOME TAX BETWEEN PERIODS \\ Walter Adolfo Noles Monteblanco* \\ Docente Auxiliar de la Facultad de Ciencias Contables \\ Universidad Nacional Mayor de San Marcos-UNMSM / Lima-Perú \\ [Recepción: Setiembre de 2014/ Conformidad: Octubre 2014]
}

\section{RESUMEN}

Los principales problemas en la contabilización del impuesto a la renta surgen del hecho que la utilidad para propósitos financieros, puede ser muy diferente a la utilidad para propósitos tributarios, con el consiguiente efecto de que el impuesto mostrado en el estado de resultados como cargo por impuesto a la renta, no guarde ninguna relación con la utilidad mostrada en dicho estado; y más aún, pudiera darse el caso, de que exista un cargo por impuesto a la renta en el estado de resultados, cuando este se encuentre mostrando una pérdida contable.

La cuestión de si el impuesto a la renta es un gasto que deba reconocerse para llegar a la utilidad neta, o es costo de hacer negocios, se enfoca en la Norma Internacional de Contabilidad $\mathrm{N}^{\circ} 12$ (NIC 12). Sin embargo, en nuestro medio este invalorable principio no tiene todavía la difusión que debería tener. Limitación que procura colaborar en superar este tratado, con la finalidad de que, como consecuencia de su comprensión y aplicación, más Estados Financieros, reflejen razonablemente la situación financiera y los resultados de sus operaciones, de conformidad a las Normas Internacionales de Información Financiera.

\section{Palabras clave:}

Impuesto a la Renta; diferencias temporarias; NIF; pérdida tributaria.

\begin{abstract}
The main problems in accounting for the income taxes arises from the fact that the utility for financial purposes, can be very different from the utility a taxable use, with the effect that the tax shown in the income statement as tax charges income, bears no relation to the value shown in that state; and further, it may be the case, that there is a charge for income tax in the income statement when this is found to show an accounting loss.

Whether the income tax is an expense that must be recognized to get to the net income or costs of doing business, focuses on the International Accounting Standard No. 12 (NIC 12). However, in our midst this invaluable principle has not yet dissemination should have. Limitation seeking to assist in overcomes this treaty, with the aim that, as a result of their understanding and application rather Financial Statements reasonably reflect the financial position and results of operations in accordance with the International Financial Reporting Standards.
\end{abstract}

\section{Keywords:}

Income Tax; Temporary differences; NIF; tax loss.

* Doctor en Ciencias Contables y Empresariales - UNMSM. Email: walter.noles@gmail.com 


\section{INTRODUCCIÓN}

El impuesto a la renta, partida de relevancia en los Estados Financieros, tanto en el estado de resultados (como cargo significativo) cuanto en el balance general (como pasivo importante relativo a los impuestos a pagar a corto plazo), nos plantea desde hace algunos años el siguiente cuestionamiento: " $₹$ Por qué ha sido mostrado durante mucho tiempo y, hasta la fecha, el monto total de impuesto a pagar (originado por la utilidad determinada de acuerdo con la legislación tributaria vigente) en el estado de resultados, si dicho estado se prepara en función de otra legislación (la legislación contable)? ¿Por qué?

Dicho impuesto, de acuerdo con los Principios de Contabilidad Generalmente Aceptados, es un gasto y como tal, debe asignarse cuando sea necesario y factible, a la utilidad del ejercicio y a las otras cuentas, en la forma que se distribuyen otros costos y gastos. Vale decir reducir la utilidad mostrando idealmente como cargo contable, el impuesto que corresponde a dicha utilidad contable. Utilidad que puede ser diferente a la tributaria, la cual servirá de base para determinar el impuesto que se pagará por dicho periodo.

Así es como, el impuesto que se muestra en el estado de resultados va a ser generalmente diferente al impuesto a pagar, si es que lo determinamos de acuerdo con el principio del periodo contable... es por esta razón, que es bueno aclarar, que este tratado tiene muy poco que ver con la preparación de las declaraciones juradas del impuesto a la renta, y con el cálculo del pasivo por este concepto a mostrar en el estado de situación financiera, de acuerdo con los dispositivos tributarios vigentes, lo cual ya está legislado.

El problema es más profundo, radica en que la distribución del impuesto a la renta entre periodos no ha sido difundido suficientemente en términos simples, no siendo aplicado en una gran mayoría de empresas donde es factible, con la finalidad de mostrar resultados contables que representen la realidad económica de la empresa; situación importante para tomar decisiones de negocios sobre bases confiables.

\section{ANTECEDENTES}

Una cuestión previa que hay que considerarse es que el impuesto a la renta, no es un costo de hacer negocios, sino, es una consecuencia de acciones u omisión de acciones de la gerencia de una empresa. Si nos inclinamos por la primera opción y el impuesto a la renta lo enfocamos como un costo de hacer negocios, lo que le cuesta a la empresa llevar a cabo sus negocios durante un ejercicio contable (determinado por la legislación vigente)- no habría asunto por resolver, y deberíamos concluir que en el estado de resultados debería mostrarse como cargo por impuesto a la renta, el monto de impuesto a pagar que muestre la declaración jurada de renta. Sin embargo, ese no es un enfoque adecuado, ya que el impuesto a la renta puede aumentar, disminuir o eliminarse, dependiendo de las decisiones gerenciales sobre el tratamiento de ciertas operaciones, las cuales tienen importantes efectos en materia de impuestos.

Como se puede observar, ya que dichos acuerdos facultan a la gerencia para decidir en qué ejercicio se va a contabilizar el impuesto a la renta, o parte del mismo, es necesario tratarlo como gasto sujeto al principio de periodicidad, con la finalidad de corregir la distorsión que podría originar en él, alguna decisión gerencial; $y$ mostrar dicho impuesto, como una variable dependiente de los resultados económicos mostrados en los Estados Financieros, para que así dichos estados, presenten razonablemente la situación financiera de la Compañía, los resultados de sus operaciones y los cambios en su situación financiera de conformidad con las Normas Internacionales de Información Financiera.

Como consecuencia de decisiones en materia de negocios, algunas transacciones afectarán ¡Hoy! la determinación de la utilidad contable, mientras que esas mismas transacciones afectarán la utilidad tributaria en otro periodo o a la inversa; originando que la utilidad para propósitos tributarios sea diferente a la utilidad para propósitos contables. Diferencias que se pueden deber a muchas causas, algunas de las cuales se exponen a continuación: 
a) Una empresa puede adoptar respecto a una transacción un método de contabilización para fines de impuestos diferente del método que sigue para fines contables, referida a esa misma transacción. Por ejemplo, una Compañía puede registrar la depreciación de un activo fijo en forma acelerada para fines contables, y emplear el método de línea recta para fines de impuestos.

b) Una partida que afecta al impuesto a la renta pudiera presentarse en el estado de cambios en el patrimonio, afectando directamente a resultados acumulados, en lugar de presentarse en el estado de resultados; como, por ejemplo, una partida de importancia que represente ingreso de ejercicios anteriores.

c) Una provisión importante por la desvalorización de inventarios es considerada contablemente como gasto en el momento de efectuar la provisión, y cuando es destruido físicamente el referido inventario, recién será considerada para efectos de impuestos.

Como en otras partidas que participan en la determinación de los resultados de operación, el problema respecto al impuesto a la renta no es cuánto registrar sino cuándo registrarlo.

No es cuánto registrar, debido a que la determinación del monto del impuesto a la renta se efectuará aplicando el dispositivo legal que se encuentre en vigencia, y que norma el asunto respecto al monto a pagar; debiendo determinarse este monto, aplicando porcentajes a la utilidad contable del ejercicio, luego de agregar o disminuir algunas partidas o aplicar incentivos o beneficios que la legislación en vigencia pudiera determinar.

El problema es cuándo registrarlo...

a) Cuando existen algunos agregados o disminuciones a la utilidad contable, los cuales son aplicados para determinar la utilidad tributaria, y que tienen impacto en el estado de situación financiera $\mathrm{La}$ NIC No 12 emitida por el Consejo de Normas Internacionales de Contabilidad (IASB), establece que se debe contabilizar el impuesto a la renta, tratando de compaginar el cargo por impuesto de un periodo con los ingresos y gastos que corresponden al mismo. b) Cuando se tiene que recuperar en el futuro un activo o liquidar en el futuro un pasivo que hoy se ha reconocido en el estado de situación financiera de la Compañía.

c) Cuando se tiene que tratar en el período corriente, la consecuencia de transacciones que han sido (o serán) objeto de reconocimiento en los estados financieros, en períodos pasados o futuros.

\section{DIFERENCIAS TEMPORARIAS}

Como lo define la NIC 12, son aquellas diferencias entre el importe en libros de un activo o pasivo determinados bajo las Normas Internacionales de Información Financiera, y su importe en el determinado, en función a la legislación tributaria vigente.

Dichas diferencias surgen cuando la época de reconocimiento de un activo o pasivo, para fines de impuestos, difiere de la época de reconocimiento para fines contables o financieros.

Las diferencias temporarias se originan en un periodo y se reversan en uno o más periodos subsecuentes, reduciendo algunas el impuesto a la renta que de otra forma, sería pagadero durante el ejercicio; otras, por el contrario, incrementan el impuesto a la renta a pagar desde el punto de vista financiero, durante el ejercicio.

Las diferencias temporarias pueden ser:

a) Diferencias temporarias imponibles, que son aquellas diferencias temporarias que dan lugar a cantidades imponibles al determinar la ganancia (pérdida) fiscal correspondiente a períodos futuros, cuando el importe en libros del activo sea recuperado o el del pasivo sea liquidado; o

b) Diferencias temporarias deducibles, que son aquellas diferencias temporarias que dan lugar a cantidades que son deducibles al determinar la ganancia (pérdida) fiscal correspondiente a períodos futuros, cuando el importe en libros del activo sea recuperado o el del pasivo sea liquidado.

Hay siete categorías de transacciones que teóricamente determinarán diferencias temporarias en un periodo; las cuales para un mejor entendimiento, serán enfocadas no solo a través del activo o pasivo, 
sino a través del impacto que causan en el resultado del ejercicio, que se muestra a continuación:

1. Diferencia entre la base contable de un activo y su base fiscal, originado por gastos o pérdidas reconocidas contablemente en un ejercicio, pero reconocidos tributariamente en ejercicios posteriores. Tales como:

a. Costos estimados de garantías por productos vendidos que se registran contablemente en el momento de la venta y se gravan impositivamente cuando se produce el desembolso.

b. Constitución de provisión para cuentas de cobranza dudosa.

c. Constitución de provisiones para desvalorización de inventarios.

2. Diferencia entre la base contable de un activo y su base fiscal, originado por gastos o pérdidas deducidas impositivamente en un ejercicio, y reconocidos contablemente en otros ejercicios posteriores, tales como:

a. Montos de depreciación en exceso de lo permitido por las disposiciones legales vigentes. Como por ejemplo; depreciación acelerada, o una forma de depreciación que origine un mayor cargo para efectos impositivos (relacionado con beneficios tributarios) y que no se consideren contablemente en ese periodo.

3. Diferencia entre la base contable de un activo y su base fiscal, originado por ingresos o gastos reconocidos contablemente en un periodo (aplicando el criterio en lo devengado) y gravados impositivamente en periodos posteriores.

4. Diferencia entre la base contable de un activo y su base fiscal, originado por ingresos gravados impositivamente en un periodo $y$ reconocidos contablemente en periodos posteriores.

5. La revaluación de activos fijos.

6. Diferencia entre activos y pasivos no monetarios, medidos en moneda funcional, versus los mismos, medidos en una moneda diferente.

\section{La pérdida tributaria arrastrable.}

La forma de hacer la distribución es el análisis de las diferencias temporarias y aplicación del efecto impositivo (el impuesto que correspondería pagar por dicha diferencia temporaria) a los activos y pasivos de los diversos periodos en los cuales se reversará dicha diferencia. Desde este punto de vista, él o los asientos por los efectos impositivos, se efectuarán en el periodo corriente, registrando las cantidades que afectarán al gasto de futuros ejercicios como activos o pasivos en el estado de situación financiera (Balance General), como se muestra en los capítulos siguientes.

\section{MÉTODO DE CONTABILIZACIÓN DEL IMPUESTO A LA RENTA}

El conocimiento de los diversos métodos de contabilización del impuesto diferido - que tiene por finalidad solucionar el problema del periodo en que debe contabilizarse este impuesto a la renta, dependiendo de la naturaleza de las diferencias que afectan su presentación, no es generalizado y menos aún, lo es su aplicación práctica en nuestro medio. En consecuencia, continúa en vigencia la alternativa de presentar en el estado de resultados el cargo por impuesto a la renta a ser pagado, que no necesariamente será el impuesto que corresponde a todas las transacciones que participaron en la determinación del resultado de operación del periodo. Continúa, digo, porque aquí se aborda este problema, y porque más allá de la forma de registro del impuesto a la renta -a la cual es indiferente el tratamiento contable de aquellos efectos impositivos que se originan en las diferencias temporarias - está el método que sí considera dicho efecto, y que distorsionan la presentación del impuesto a la renta en lo que a periodicidad y correlación se refiere. Método que será analizado y ejemplarizado, con el fin de que el cargo por impuesto a la renta sea representativo de todas las transacciones que determinaron el resultado de operación del presente ejercicio económico.

El método para llevar a cabo dicho propósito es expuesto a continuación: 


\section{DISTRIBUCIÓN DEL IMPUESTO ALA RENTA ENTRE PERÍODOS BAJO EL MÉTODO DEL PASIVO BASADO EN EL ESTADO DE SITUACIÓN FINANCIERA}

Ala luz de este método se aplican procedimientos de contabilización de impuestos por medio de los cuales, el efecto impositivo que originan las diferencias temporarias (el impuesto que le corresponde a tal diferencia) es diferido en una cuenta de activo o pasivo del estado de situación financiera; $y$ asignado al cargo, por impuesto a la renta del periodo.

Este tratamiento contable está diseñado para reflejar en el cargo por impuesto a la renta el efecto impositivo de la diferencia temporaria en el año, en el cual ella se originó. Por ejemplo, el hecho de constituir una provisión en el estado de situación financiera con su contrapartida en gastos, sea para cuentas de cobranza dudosa o para desvalorización u obsolescencia de inventarios, genera un cargo contable en el estado de resultados que no es aceptado para efectos tributarios en ese año.

La base contable de esta provisión será el monto que aparece en el activo del balance. Sin embargo, para fines de impuesto, la base fiscal será (0).

Dicho de otra forma, este cargo originará una diferencia temporaria, ya que dicha provisión tendrá que agregarse hoy (deducirse después) a la utilidad contable determinada en un ejercicio, para llegar a la utilidad tributaria y calcular el impuesto, el cual irá en el estado de resultados como deducción de la utilidad contable, con lo cual tendremos un impuesto en el estado de resultados que no guardará correlatividad con todos los gastos e ingresos, pues hay una partida (la referida provisión) que no es de este año, para efecto de impuestos.

Con la aplicación del método distribución del impuesto a la renta entre períodos, pretendemos corregir esta diferencia en la correlatividad originada por aquellas diferencias temporarias.

Los mayores o menores impuestos contables (impuestos diferidos) están determinados sobre la base de las tasas de impuestos vigentes para el momento en que se originaron las diferencias temporales, y no se ajustan subsecuentemente por cambio en las tasas de impuestos o para reflejar nuevos impuestos, hasta que este impuesto sea compensado.

Asimismo, el efecto que surge de aplicar la tasa de impuesto sobre las diferencias temporarias -las cuales reducen el cargo por impuesto a la renta- es tratado como cargo (activo) diferido en la cuenta del activo; y por otro lado, el efecto que surge de aplicar la tasa de impuesto sobre las diferencias temporarias -las cuales aumentan el cargo por impuesto a la renta- es tratado como crédito (pasivo) diferido en las cuentas del pasivo. La reversión de estos efectos impositivos (mayores o menores impuestos a la renta contables), sean débitos o créditos, se efectúa aplicando dichas diferencias al cargo por impuesto a la renta futuro, $y$ la forma de su aplicación, está basada en la manera en la cual las mismas diferencias temporarias, participan en la determinación de la utilidad de operación en relación con la renta imponible en dichos periodos.

\section{DESARROLLO PRÁCTICO SOBRE EL TRATAMIENTO DE EFECTOS IMPOSITIVOS}

Quiero remarcar, antes de exponer algunos casos típicos sobre el tratamiento que debe darse a algunas diferencias temporarias en el Perú, que el control sobre el cumplimiento en el pago de los impuestos, aun no siendo tan frecuente en nuestros negocios, tiene un significado especial, ya que el mismo debe hacerse de acuerdo con las disposiciones tributarias, para así minimizar los riesgos de mayores desembolsos relacionados con penalidades por incumplimiento.

Consideramos que aun siendo numerosas las operaciones sobre impuestos importantes -lo cual nos permite cierta holgura en el tiempo para su procedimiento y menor riesgo de omisión- los errores que muchas veces se encuentran, están ubicados en el adecuado cálculo, desde el punto de vista tributario, lo cual se elimina con un buen conocimiento de la legislación vigente sobre los mismos.

Sin embargo, debemos tener presente que a través del proceso de este texto, y con la finalidad que se 
persigue (que sus estados financieros sean expuestos razonablemente, de acuerdo con las Normas Internacionales de Información Financiera), nuestro objetivo es efectuar una conciliación entre el resultado del cálculo que se ha obtenido como consecuencia de aplicar las disposiciones tributarias vigentes, con el resultado obtenido al aplicar los principios contables; así como, el análisis del tratamiento de las diferencias que por lo general, siempre existen.

Con ese fin, y como apoyo a lo expuesto, se expresa a continuación algunos casos prácticos relacionados con las partidas que afectan financieramente el cargo por impuesto a la renta y la distribución del mismo, entre los diferentes periodos contables en que estas partidas tienen vigencia.

Debe tenerse en consideración que los casos prácticos, las soluciones y el desarrollo de los mismos que aquí se exponen son incluidos para efectos enunciativos y con la finalidad de apreciar las virtudes que persigue este tratado. Es así que, aun cuando la legislación tributaria en el país no se puede considerar estable, en su esencia el problema que origina en los Estados Financieros siempre tendrá vigencia, dependiendo del tipo de diferencia que se esté tratando entre la legislación financiera y la legislación tributaria.

En conclusión, este trabajo es aplicable en su concepto para cualquier periodo contable, pasado, presente, $y$ en adelante.
DIFERENCIAS ENTRE LA BASE CONTABLE DE UN ACTIVO Y SU BASE FISCAL, ORIGINADAS POR GASTOS RECONOCIDOS CONTABLEMENTE ENUN EJERCICIO, PERO RECONOCIDAS TRIBUTARIAMENTE EN EJERCICIOS POSTERIORES

\section{Caso 1.- Provisión para desvalorización de in- ventarios}

Durante el ejercicio de 2012 el gerente general de la empresa comercial EL INVENTO (constituida como Sociedad Anónima) acuerda efectuar una provisión para desvalorización del 100\%, para aquellos inventarios que tienen una antigüedad mayor a un año, lo cual obedece a política interna, por constantes renovaciones tecnológicas de los productos que fabrican. Debido a lo cual, transcurrido algún tiempo, se hacen de dificultosa realización. Estos inventarios ascienden a $\mathrm{S} / .500,000$, los cuales fueron provisionados con cargos de gastos administrativos y con abono a la provisión para desvalorización de inventario.

Como consecuencia de esta provisión, la utilidad, antes del impuesto a la renta, se redujo en dicho importe. La Compañía efectuó trámites pertinentes para eliminar el inventario provisionado, para lo cual previamente se reunió el Directorio y acordó lo siguiente:

"Debido a las características del producto, la destrucción se efectuará en una cantidad determinada mensual, ajustándose a las exigencias de ley, para que el monto de la destrucción sea aceptado como un gasto deducible para efectos tributarios".

De acuerdo a la normatividad tributaria en el Perú, estas reservas no podrán ser deducidas como gasto, hasta que no se compruebe notarialmente la eliminación física del inventario. Efectuando un cálculo de la cantidad que se destruirá mensualmente, se estimó que el inventario provisionado se destruiría en un total de 10 meses, de enero a octubre de 2013.

Los estados de resultados de los años 2012 y 2013 se presentan como sigue: 
Estados de Resultados

\begin{tabular}{|c|c|c|c|}
\hline & 2012 & 2013 & Total \\
\hline & $\mathrm{S} /$. & $\mathrm{S} /$. & $\mathrm{S} /$. \\
\hline Utilidad operativa & $1 ’ 000,000$ & 1’000,000 & 2’000,000 \\
\hline $\begin{array}{l}\text { Provisión para desvalorización de } \\
\text { inventarios }\end{array}$ & $(500,000)$ &.-- & $(500,000)$ \\
\hline Utilidad antes de impuesto a la renta & 500,000 & $1^{\prime} 000,000$ & 1'500,000 \\
\hline Impuesto a la renta $40 \%\left({ }^{*}\right)$ & $(400,000)$ & $(200,000)$ & $(600,000)$ \\
\hline \multicolumn{2}{|r|}{$80 \%$} & $20 \%$ & $40 \%$ \\
\hline Utilidad Neta & 100,000 & 800,000 & 900,000 \\
\hline
\end{tabular}

$\left.{ }^{*}\right)$ El impuesto a la renta fue obtenido de las Declaraciones Juradas que siguen a continuación:

Declaraciones Juradas

\begin{tabular}{|c|c|c|c|}
\hline & 2012 & 2013 & Total \\
\hline & $S /$. & $S /$. & $S /$. \\
\hline Utilidad según balance & 500,000 & $1^{\prime} 000,000$ & 1'500,000 \\
\hline $\begin{array}{l}\text { Adición (deducción) de la provisión } \\
\text { para desvalorización de inventarios }\end{array}$ & 500,000 & $(500,000)$ &.-- \\
\hline Utilidad imponible & 1’000,000 & 500,000 & 1'500,000 \\
\hline Impuesto a la renta a pagar $40 \%$ & 400,000 & 200,000 & 600,000 \\
\hline
\end{tabular}

Si analizamos el estado de resultados podremos apreciar que en el año 2012, la utilidad contable que ascendió a S/. 500,000 fue gravada con S/.400,000 de impuesto a la renta, representando el $80 \%$ de esta utilidad; mientras que en el año 2013, habiendo obtenido una utilidad de S/. 1'000,000 el impuesto que se pagó fue de 200,000, representando el $20 \%$ de dicha utilidad.

¿Por qué el año 2012 el impuesto representa el $80 \%$ y el año 2013 sólo el $20 \%$ de la utilidad?

Si analizamos esta distorsión, llegaremos a la conclusión que la diferencia de los porcentajes de impuestos es producto del adelanto de registro contable de una partida que, para efectos tributarios, se considerará como gasto en otro periodo y que, en consecuencia, origina que no se logre una adecuada correlación de ingresos con gastos entre dichos ejercicios.

La aplicación del principio de distribución de impuestos a la renta entre periodos, para este caso, permitiría que la Compañía muestre una adecuada correlación de ingresos con gastos, de la forma, como se expone a continuación: 
Estados de Resultados

\begin{tabular}{|c|c|c|c|}
\hline & 2012 & 2013 & Total \\
\hline & S/. & $\mathrm{S} /$. & $S /$. \\
\hline Utilidad operativa & $1 ’ 000,000$ & 1’000,000 & $2 ’ 000,000$ \\
\hline $\begin{array}{l}\text { Provisión para desvalorización de } \\
\text { inventarios }\end{array}$ & $(500,000)$ &.-- & $(500,000)$ \\
\hline Utilidad antes de impuesto a la renta & 500,000 & 1'000,000 & $1 ' 500,000$ \\
\hline Impuesto a la renta $40 \%\left({ }^{*}\right)$ & 400,000 & 200,000 & 600,000 \\
\hline $\begin{array}{l}\text { Efectos impositivos de las diferencias } \\
\text { temporales }\end{array}$ & $(200,000)$ & 200,000 &.-- \\
\hline Cargo por impuesto a la renta & 200,000 & 400,000 & 600,000 \\
\hline \multicolumn{2}{|r|}{$40 \%$} & $40 \%$ & $40 \%$ \\
\hline Utilidad Neta & 300,000 & 600,000 & 900,000 \\
\hline
\end{tabular}

A continuación, se presentan los asientos contables que deben realizarse con la finalidad de corregir la distorsión que origina, en el impuesto a la renta, el efecto impositivo de la diferencia temporaria:

Año 2012

\begin{tabular}{|c|c|c|}
\hline & Cargo & Abono \\
\hline $\begin{array}{l}\text { DR. Impuesto a la renta } \\
\text { (Cuenta de resultados) }\end{array}$ & 400,000 & \\
\hline $\begin{array}{l}\text { CR. Impuestos por pagar } \\
\text { (Cuenta de balance) }\end{array}$ & & 400,000 \\
\hline
\end{tabular}

Por el cargo normal a resultados, determinado en la declaración jurada de renta del año 2012.

----------- 2 ------------

DR. Impuesto a la renta diferido $\quad 200,000$

(Cuenta de balance)

200,000

CR. Impuesto a la renta

(Cuenta de resultados)

Para regularizar el mayor cargo contable a resultados por impuesto a la renta, afectando al balance (activo diferido) el efecto impositivo que origina la diferencia temporaria y resulta de aplicar $40 \%$ (Tasa de impuesto) a la provisión para desvalorización (Diferencia entre la base contable y la base tributaria del rubro de inventarios) del balance general.
Se podrá observar, en consecuencia, que el cargo por impuesto a la renta, que ascendía a $S / .400,000$ y que equivale en el primer asiento a la tasa de $80 \%$, de la utilidad contable es el que se pagará al fisco según la declaración jurada de renta. Sin embargo, de ese monto, $S / .200,000$ serán desplazados hacia una cuenta de activos diferidos de la empresa, deduciendo contablemente el cargo a resultados por impuesto a la renta, lo cual es representativo de aplicar a resultados del ejercicio una tasa de impuesto de $40 \%$ a la partida $\mathrm{S} / .500,000$ que está incluido en él, y que representa la utilidad antes del impuesto.

Para el año siguiente (2013) asumiremos que dicha partida que se provisionó el año anterior sí es aceptada como gasto, debido a que se efectuó la destrucción de la mercadería, cumpliendo todos los trámites notariales que para efecto de su aceptación ella requiere.

\section{Año 2013}

El impuesto que deberá pagarse ese año es de S/.200,000, según la declaración jurada de renta; lo cual difiere del cargo contable por impuesto a la renta cargable a resultados, que es de S/.400,000.

Para efecto de una adecuada presentación de la correlación, ingresos con gastos, se deberá mostrar como cargo de impuesto a la renta S/.400,000, lo cual se logra efectuando los siguientes asientos contables: 
Cargo Abono $-3$

DR. Impuesto a la renta

(Cuenta de resultados)

CR. Impuestos por pagar

(Cuenta de balance)

Por el cargo normal a resultados, determinado en la declaración jurada de renta del año 2013. $4-$

DR. Impuesto a la renta

(Cuenta de balance)

200,000

CR. Impuesto a la renta diferido

(Cuenta de resultados)

200,000

Para mostrar adecuadamente el cargo a ganancias y pérdidas por impuesto a la renta, reversando el asiento efectuado el año anterior por el efecto impositivo de la diferencia temporaria.
Es necesario remarcar que, si bien el estado de resultados incluye un impuesto a la renta que tiene correlación con todos los ingresos y gastos que allí se muestran, no necesariamente debe tener coincidencia con el impuesto que se abonará a la Administración tributaria, y por lo tanto, tampoco será igual al pasivo por impuesto mostrado en el estado de situación financiera.

Dicha diferencia, de existir, se estará mostrando en una partida diferente del Balance General, en el activo o en el pasivo (activo (pasivo) por impuesto diferido), lo cual representará el efecto impositivo de las diferencias temporarias que fueron las motivadoras de este principio contable de distribución del impuesto a la renta entre periodos; de la forma como resumidamente se expone a continuación:

Estados de Resultados

\begin{tabular}{|c|c|c|c|}
\hline & $\mathbf{2 0 1 2}$ & $\mathbf{2 0 1 3}$ & Total \\
\cline { 2 - 4 } & $\mathrm{S} /$. & $\mathrm{S} /$. & $\mathrm{S} /$. \\
\hline Utilidad antes de impuesto a la renta & 500,000 & 1'000,000 & 1'500,000 \\
\hline Cargo por Impuesto a la renta (Nota 1) & 200,000 & 400,000 & 600,000 \\
\hline Porcentaje & $\mathbf{4 0 \%}$ & $\mathbf{4 0 \%}$ & $\mathbf{4 0 \%}$ \\
\hline
\end{tabular}

NOTA 1:

\begin{tabular}{|l|c|c|}
\hline & $\mathbf{2 0 1 2}$ & $\mathbf{2 0 1 3}$ \\
\hline Impuesto a la renta por pagar & 400,000 & 200,000 \\
\hline Impuesto a la renta diferido & $(200,000)$ & 200,000 \\
\hline Total cargo por impuesto a la renta & $\mathbf{2 0 0 , 0 0 0}$ & $\mathbf{4 0 0 , 0 0 0}$ \\
\hline
\end{tabular}

DIFERENCIAS ENTRE LA BASE FISCAL Y LA BASE CONTABLE DE UN ACTIVO, ORIGINADAS POR GASTOS O PÉRDIDAS DEDUCIDAS PARA FINES TRIBUTARIOS EN UN EJERCICIO Y RECONOCIDAS CONTABLEMENTE EN EJERCICIOS POSTERIORES

\section{Caso 2.- Depreciación acelerada}

Acatando disposiciones legales, y con la finalidad de obtener beneficios tributarios, la Compañía, Actifi S.A. durante el año 2009, adquirió algunos activos fijos en el mes de diciembre de ese año. Dicho beneficio consistía en que podrían ser depreciados en
3 años a razón del 33\% anual para fines tributarios; aun cuando la vida útil del activo se estima en cinco años; lo cual, prescindiendo del valor residual que al final pudiera tener (el cual era considerado cero), representaba una tasa de depreciación contable de $20 \%$.

La gerencia de la Compañía Actifi S.A. (Compañía que adquirió dichos activos). Al decidir al respecto, consideró que lo más conveniente para la empresa era depreciar dichos activos fijos en cinco años para efectos contables, esto es, depreciarlos en una tasa del $20 \%$ anual; y en forma acelerada para efectos tributarios, con la finalidad de gozar de los 
beneficios de reducción de la materia imponible que dicho procedimiento traía consigo en los primeros años, lo cual les era más conveniente para efectos de llevar a cabo los planes de reinversión.

El costo de dichos activos fijos ascendía a S/.1'500,000, por los cuales la depreciación tributaria ascendía a $S / .500,000$ anuales; y la depreciación contable ascendía a S/.300,000 anuales. Las utilidades contables que la Compañía Actifi S.A. obtuvo, antes de contabilizar las correspondientes depreciaciones durante los años que se mantuvo contablemente este activo, fueron las siguientes:

$\begin{array}{ll}\text { Año 2009 } & \text { 1'500,000 } \\ \text { Año 2010 } & \text { 1'500,000 } \\ \text { Año 2011 } & \text { 1'500,000 } \\ \text { Año 2012 } & \text { 1'500,000 } \\ \text { Año 2013 } & \text { 1'500,000 }\end{array}$

Los Estados de resultados de la Compañía por los años antes mencionados fueron los siguientes:

Efectuando un análisis de la situación aquí presentada, podremos advertir lo siguiente:
En los Estados de Resultados hemos consignado la utilidad que la Cía. Actifi S.A. obtuvo durante los años 2009 a 2013, mostrando en forma separada la depreciación anual, no diferenciando de la misma el cargo a gastos o costos de producción (alternativa que es indiferente en este caso), para efecto de un mejor análisis.

La utilidad contable antes de impuesto se presenta, como usted puede observar, uniforme, la que asciende a $S / .1,200,000$ cada año, y sobre la cual se debe pagar un impuesto que se muestra allí en la siguiente línea. Pero, a diferencia de la uniformidad que muestra la utilidad contable, el impuesto que debe pagarse cada año no lo es. Note usted que se pagará un impuesto similar de S/.500,000 durante los tres primeros años, el cual sufre una variación significativa el cuarto y quinto año, la que está originada por la diferencia en el tratamiento de la depreciación para efectos tributarios.

Estados de Resultados

\begin{tabular}{|l|c|c|c|c|c|c|}
\hline & $\mathbf{2 0 0 9}$ & $\mathbf{2 0 1 0}$ & $\mathbf{2 0 1 1}$ & $\mathbf{2 0 1 2}$ & $\mathbf{2 0 1 3}$ & Total \\
\cline { 2 - 8 } & $\mathrm{S} /$. & $\mathrm{S} /$. & $\mathrm{S} /$. & $\mathrm{S} /$. & $\mathrm{S} /$. & $\mathrm{S} /$. \\
\hline Utilidad operativa & $1,500,000$ & $1,500,000$ & $1,500,000$ & $1,500,000$ & $1,500,000$ & $7,500,000$ \\
\hline Depreciación del año & $(300,000)$ & $(300,000)$ & $(300,000)$ & $(300,000)$ & $(300,000)$ & $1,5000,000$ \\
\hline & & & & & & \\
\hline $\begin{array}{l}\text { Utilidad contable antes } \\
\text { de impuestos }\end{array}$ & $\mathbf{1 , 2 0 0 , 0 0 0}$ & $\mathbf{1 , 2 0 0 , 0 0 0}$ & $\mathbf{1 , 2 0 0 , 0 0 0}$ & $\mathbf{1 , 2 0 0 , 0 0 0}$ & $\mathbf{1 , 2 0 0 , 0 0 0}$ & $\mathbf{6 , 0 0 0 , 0 0 0}$ \\
\hline Impuesto a la renta 50\% $\left(^{*}\right)$ & $(500,000)$ & $(500,000)$ & $(500,000)$ & $(750,000)$ & $(750,000)$ & $3,0000,000$ \\
\hline & & & & & & \\
\hline
\end{tabular}

${ }^{*}$ ) Se puede observar que aun cuando la base general corresponde al 50\%, los cargos por impuesto a la renta, no corresponden a la mitad de la utilidad contable. El impuesto a la renta fue obtenido de las declaraciones juradas que se presentan a continuación: 


\section{Declaración Jurada}

\begin{tabular}{|l|c|c|c|c|c|c|}
\hline & $\mathbf{1 9 7 9}$ & $\mathbf{1 9 8 0}$ & $\mathbf{1 9 8 1}$ & $\mathbf{1 9 8 2}$ & $\mathbf{1 9 8 3}$ & Total \\
\cline { 2 - 7 } & $\mathrm{S} /$. & $\mathrm{S} /$. & $\mathrm{S} /$. & $\mathrm{S} /$. & $\mathrm{S} /$. & $\mathrm{S} /$. \\
\hline $\begin{array}{l}\text { Utilidad contable } \\
\text { antes de deprecia- } \\
\text { ciones }\end{array}$ & $1,200,000$ & $1,200,000$ & $1,200,000$ & $1,200,000$ & $1,200,000$ & $6,000,000$ \\
\hline $\begin{array}{l}\text { Depreciación } \\
\text { contable }\end{array}$ & 300,000 & 300,000 & 300,000 & 300,000 & 300,000 & $1,5000,000$ \\
\hline & & & & & & \\
\hline $\begin{array}{l}\text { Utilidad } \\
\text { operativa }\end{array}$ & $\mathbf{1 , 5 0 0 , 0 0 0}$ & $\mathbf{1 , 5 0 0 , 0 0 0}$ & $\mathbf{1 , 5 0 0 , 0 0 0}$ & $\mathbf{1 , 5 0 0 , 0 0 0}$ & $\mathbf{1 , 5 0 0 , 0 0 0}$ & $\mathbf{7 , 5 0 0 , 0 0 0}$ \\
\hline $\begin{array}{l}\text { Deducción de la } \\
\text { depreciación } \\
\text { tributaria }\end{array}$ & $(500,000)$ & $(500,000)$ & $(500,000)$ & & & \\
\hline
\end{tabular}

Observemos la declaración jurada

La utilidad imponible (utilidad sobre la cual se calcula el impuesto a la renta) ha sido determinada dejando sin efecto la depreciación ya registrada contablemente, para lo cual se adiciona a la utilidad operativa, no aceptando como gasto, los S/.300,000 mensuales que se cargaron durante los años en que contablemente dicho activo tuvo vida útil, $\mathrm{y}$ computando la depreciación de dicho activo en tres años; esto es, restando S/.500,000 a dicha utilidad durante los tres primeros años, con la finalidad de gozar del beneficio tributario, al tener menos utilidad tributaria para pagar menos impuestos.

Como usted podrá observar, tributariamente el impuesto a la renta será siempre el 50\%, estando la diferencia en los montos del impuesto a pagar originados por la variación en la utilidad imponible, por lo cual aun cuando contablemente la utilidad financiera es un mismo importe, el impuesto a la renta que se aplicará como cargo en el estado de resultados es diferente.

Esta distorsión está originada por la diferencia en el tratamiento de la depreciación entre el aspecto contable y el aspecto tributario; diferencia que ha sido expuesta en los cuadros anteriores y que vista desde la óptica del rubro del activo, será la diferencia entre la base contable y la base fiscal para el rubro activo fijo; que origina una diferencia temporaria.

La corrección de la misma sólo se logrará aplicando el principio de la distribución del impuesto a la renta entre períodos, con lo cual se logrará una adecuada correlación de ingresos con gastos; eliminando en este estado el efecto contable que causa el adelanto de registro de la depreciación para efectos tributarios, de la forma como se muestra a continuación: 
Estados de Resultados

\begin{tabular}{|l|c|c|c|c|c|c|}
\hline & $\mathbf{2 0 0 9}$ & $\mathbf{2 0 1 0}$ & $\mathbf{2 0 1 1}$ & $\mathbf{2 0 1 2}$ & $\mathbf{2 0 1 3}$ & Total \\
\cline { 2 - 7 } & $\mathrm{S} /$. & $\mathrm{S} /$. & $\mathrm{S} /$. & $\mathrm{S} /$. & $\mathrm{S} /$. & S $/$. \\
\hline Utilidad operativa & $1,500,000$ & $1,500,000$ & $1,500,000$ & $1,500,000$ & $1,500,000$ & $7,500,000$ \\
\hline Depreciación del año & $(300,000)$ & $(300,000)$ & $(300,000)$ & $(300,000)$ & $(300,000)$ & $1,5000,000$ \\
\hline & & & & & & \\
\hline $\begin{array}{l}\text { Utilidad antes de } \\
\text { impuesto }\end{array}$ & $\mathbf{1 , 2 0 0 , 0 0 0}$ & $\mathbf{1 , 2 0 0 , 0 0 0}$ & $\mathbf{1 , 2 0 0 , 0 0 0}$ & $\mathbf{1 , 2 0 0 , 0 0 0}$ & $\mathbf{1 , 2 0 0 , 0 0 0}$ & $\mathbf{6 , 0 0 0 , 0 0 0}$ \\
\hline Impuesto a la renta & 500,000 & 500,000 & 500,000 & 750,000 & 750,000 & $3,0000,000$ \\
\hline $\begin{array}{l}\text { Efectivo impositivo de } \\
\text { la diferencia } \\
\text { temporaria }\end{array}$ & 100,000 & 100,000 & 100,000 & $(150,000)$ & $(150,000)$ &.-- \\
\hline & & & & & & \\
\hline & $\mathbf{6 0 0 , 0 0 0}$ & $\mathbf{6 0 0 , 0 0 0}$ & $\mathbf{6 0 0 , 0 0 0}$ & $\mathbf{6 0 0 , 0 0 0}$ & $\mathbf{6 0 0 , 0 0 0}$ & $\mathbf{3 , 0 0 0 , 0 0 0}$ \\
\hline & & & & & & $\mathbf{6 0 0 0}$ \\
\hline Utilidad Neta & $\mathbf{6 0 0 , 0 0 0}$ & $\mathbf{6 0 0 , 0 0 0}$ & $\mathbf{6 0 0 , 0 0 0}$ & $\mathbf{6 0 0 , 0 0 0}$ & $\mathbf{6 0 0 , 0 0 0}$ & $\mathbf{3 , 0 0 0 , 0 0 0}$ \\
\hline
\end{tabular}

A continuación, se presentan los asientos contables que deben efectuarse para corregir el efecto distorsionante que origina la diferencia de periodicidad en el registro contable y tributario de la depreciación de activos fijos sobre los Estados Financieros que determina una diferencia entre la base fiscal y la base contable de este rubro en el estado de situación financiera a través de la cuenta depreciación acumulada.

Los asientos serían los siguientes:

Año 2009

\begin{tabular}{|c|c|c|}
\hline & Cargo & Abono \\
\hline $\begin{array}{l}\text { DR. Impuesto a la renta } \\
\text { (Cuenta de resultados) }\end{array}$ & 500,000 & \\
\hline $\begin{array}{l}\text { CR. Impuestos por pagar } \\
\text { (Cuenta de balance) }\end{array}$ & & 500,000 \\
\hline
\end{tabular}

Impuesto a la renta

\begin{tabular}{lll}
2009 & 500,000 & $\begin{array}{l}\text { Se cancela contra el } \\
\text { resultado de opera- } \\
\text { ciones en el mismo }\end{array}$ \\
& 100,000 & año. \\
\hline-------- &
\end{tabular}

Impuesto a la renta

\begin{tabular}{|c|c|c|}
\hline 2010 & a) 500,000 & Se cancela contra el \\
\hline 2010 & 100,000 & $\begin{array}{l}\text { resultado de opera- } \\
\text { ciones en el mismo }\end{array}$ \\
\hline
\end{tabular}

Por el cargo a resultados, determinado según la declaración jurada de impuesto a la renta.

$$
2
$$

DR. Impuesto a la renta diferido $\quad 100,000$ (Cuenta de resultados)

CR. Impuesto a la renta

(Cuenta de pasivo diferido)

100,000

Por el mayor cargo a resultados que corresponde al efecto impositivo de la diferencia temporaria, el cual tiene por finalidad efectuar una adecuada correlación de ingresos con gastos.

Se efectuaran asientos similares, uno con la finalidad de contabilizar el impuesto que se determine según la declaración jurada, y otro con la finalidad de corregir la distorsión originada por el adelanto en el registro tributario de la depreciación del activo fijo, obteniéndose los siguientes saldos:

\section{Impuestos por pagar}

Se cancela en el año

2010 por el pago con

abono a bancos.

Impuesto a la renta diferido

\section{Impuestos por pagar}

Se cancela en 1981 por

el pago con abono a ban-

cos.

Impuesto a la renta diferido a) 500,000

b) 600,000

2010

500,000

2010

100,000 
La cuenta de impuestos por pagar se saldará cuando se efectúe el pago. Nótese que aun cuando el impuesto anual mostrado en el estado de resultado es S/.600,000 cada uno de estos años, se cancelará al fisco el impuesto correcto, vg. el determinado según la Declaración Jurada.

\begin{tabular}{lll}
\multicolumn{3}{r}{ Impuesto a la renta } \\
$2011 \quad 500,000$ & $\begin{array}{l}\text { Se cancela contra el } \\
\text { resultado de opera- } \\
\text { ciones. }\end{array}$
\end{tabular}

\begin{tabular}{lr} 
& \\
& $-100,000$ \\
\hline 2009 & 100,000 \\
2010 & 100,000 \\
2011 & 100,000
\end{tabular}

Saldos acumulados durante los tres años mencionados

\section{Impuestos por pagar}

Se cancela en 2012 con

abono a bancos.

Impuesto a la renta diferido
2011

500,000

2010

100,000

$\underline{600,000}$
Habiendo llegado al tercer año - año en el que se trasladaba tributariamente el costo del activo fijo a resultados, y habiendo reflejado el efecto que origina en resultados la diferencia en los períodos de contabilización durante los tres primeros años; con la finalidad de continuar con la uniformidad que debe haber entre los ingresos y gastos, debemos tomar en cuenta el saldo de pasivo diferido, en el balance, y comenzar a reversarlo con abono a ganancias y pérdidas, en la medida que el estado de resultados, desde el punto de vista contable, se vea afectado por los cargos que se harán por la depreciación del activo que aún se sigue efectuando contablemente. Los asientos serían los siguientes:

Año 2012

$\begin{aligned} & \text { DR. Impuesto a la renta } \\ & \text { (Cuenta de resultados) }\end{aligned}$
$\begin{aligned} & \text { CR. Impuestos por pagar } \\ & \text { (Cuenta de pasivos) }\end{aligned}$

700,000

Por el cargo a resultados, determinado según la Declaración Jurada de Impuesto a la Renta.

DR. Impuesto a la renta diferido $\quad 150,000$
(Cuenta de pasivo diferido)
CR. Impuestos a la renta
(Cuenta de resultados)

150,000

Por el menor cargo a resultados que corresponde al efecto impositivo de la diferencia temporaria por haber adelantado el registro tributario de la depreciación del activo fijo.
Como se podrá observar, durante el ejercicio 2012 el Impuesto a la Renta que deberá pagarse es de S/.750,0000, el cual surge de la Declaración Jurada, y es mayor que el año anterior $(S / .500,000)$ debido a que durante los años 2012 y 2013 no habrá cargo por la depreciación del activo fijo en cuestión, en razón de que tributariamente ya no tiene vida útil. Sin embargo, la utilidad contable (S/. 1'200,000) es diferente a la utilidad tributaria (S/. 1'500,000) debido a que contablemente dicho activo aún tiene vida útil, y por el cual se ha cargado en resultados S/.300,000 por concepto de depreciación; por lo tanto, el impuesto que le corresponde a dicha utilidad contable incluye una partida de gastos que fue considerada para determinar la utilidad tributaria y pagar impuesto al año anterior, ocasionando una distorsión en el cargo que idealmente debe aparecer mostrado como Impuesto a la Renta en el estado de resultados.

Esta distorsión la corregimos descargando de la cuenta Impuesto a la Renta Diferido, la porción correspondiente; e incrementando, por el contrario, el cargo a resultados por Impuesto a la Renta en S/. 150,000. Cabe mencionar, que para el año 2013 los asientos que deben efectuarse serían los mismos; con el siguiente efecto: (1) quedando saldada la cuenta Impuesto a la Renta Diferido, (2) habiendo pagado el fisco, cada añolos impuestos que realmente correspondieron a la utilidad tributaria y (3) logrando mostrar, durante cada uno de los cinco años, una 
adecuada correlación entre la utilidad contable del período y el cargo a resultados por Impuesto a la Renta.

\section{LA PÉRDIDA TRIBUTARIA ARRASTRABLE}

Las pérdidas anuales que genera un negocio determinado pueden ser de tipo contable o tributario. Contable son las que con frecuencia son llamadas pérdidas económicas, y son aquellas que se obtienen en los Estados Financieros de la Compañía, los que generalmente, son preparados de acuerdo con principios y/o prácticas contables. Tributarias son aquellas que se obtienen de acuerdo con criterios tributarios; es decir, que partiendo de la utilidad o pérdidas económicas, se le efectúan algunas correcciones de operaciones o diminuciones a dicho monto, dando lugar en muchos casos a un nuevo monto que será la utilidad o pérdida tributaria.

Dicha pérdida tributaria tiene un tratamiento especial para efectos fiscales, ya que podrán ser deducidas de las utilidades tributarias los siguientes cuatro años, reduciendo, así, la base o materia imponible y, consecuentemente, el impuesto a pagar en dichos ejercicios. Vale decir, el impuesto que deberá pagarse será menor que el que hubiera correspondido de no existir beneficio tributario (ahorro de impuesto) que da la ley a través de la aplicación de las pérdidas tributarias que se arrastran para el futuro. Surge entonces la pregunta siguiente: ¿debe conocerse el beneficio tributario futuro de pagar menos impuestos en el año en que se originó dicha pérdida tributaria?

El reconocer dicho beneficio implica que la empresa es normalmente rentable, y que la pérdida que se ha obtenido es originada por algún hecho fortuito, no recurrente.
Debe evaluarse como condición previa estas dos alternativas y la situación futura de la empresa, con la finalidad de asegurar la aplicación total futura del beneficio tributario obtenido en determinado año.

No debe asombrar el hecho de tener pérdida contable y, luego de ciertos agregados o disminuciones a dicho monto, obtener utilidad tributaria, y consecuentemente impuesto a la renta.

El beneficio futuro que origina dicha pérdida tributaria, a consecuencia de partidas que puedan ser consideradas diferencias temporarias, puede mostrarse en el estado de resultados, afectando el Impuesto a la Renta.

Quiere decir esto, que aun teniendo pérdida contable existe Impuesto a la Renta Negativo, que será el reconocimiento en este año del beneficio futuro que ocasionará dicha partida.

Cabe mencionar que, en el año en que se produzca una pérdida tributaria originada por una diferencia permanente no existirá relación entre el impuesto y dicha pérdida a nivel porcentual. Sin embargo, cuando la pérdida tributara sea originada por diferencias temporarias, debido a que existe la posibilidad de recuperar el efecto tributario, tanto por la pérdida tributaria como por la diferencia temporaria, conviene diferir el efecto neto entre la diferencia temporaria y la pérdida tributaria.

Mostramos a continuación los siguientes casos: 1. P.T. v.s. Útil contable originada por $\neq$ permanente.

2. P.T. v.s. PC originada por $\neq$ permanente.

3. P.T. v. UC originada por diferencia temporal. 


\section{Declaración Jurada}

Utilidad (pérdida) contable

Gastos no aceptados

Provisión a ser reversada el año siguiente

\section{Pérdida tributaria}

\section{Beneficios tributarios}

Por la pérdida tributaria $40 \%$

Por la diferencia temporaria $40 \%$

\section{Total beneficio tributario diferido mayor} (menor) impuesto

\section{Estado de Resultados (corregido)}

Utilidad (pérdida) contable

Impuesto a la Renta (beneficio tributario) 40\%

$$
\text { Utilidad (pérdida) neta }
$$

\section{REVELACIÓN EN LOS ESTADOS FINANCIEROS}

Como se ha podido observar, a través de lo expresado anteriormente, la aplicación del principio de distribución de impuesto a la renta entre períodos, origina efectos tributarios que se deben diferir para constituir más, o menos impuestos en el futuro. Este efecto puede ser tanto activo como pasivo.

Considero que es necesario identificar la situación de la partida que origina la diferencia entre la base contable y la base fiscal, y que causa el efecto tributario, con la finalidad de darle una adecuada clasificación a dicho efecto. Si se han constituido partidas que afectaron la situación corriente de la Compañía (como por ejemplo provisión para obsolescencia o desvalorización de inventarios).

Considero razonable que el efecto impositivo que se diferirá deberá clasificarse dentro del activo o pasivo corriente del estado de situación financiera.

En el caso contrario, que esté originado por depreciación acelerada, $u$ otro que sea de origen no corriente, debería ser clasificado como activo o pasivo no corriente.

$\begin{array}{ccc}\text { CASO 1 } & \text { CASO 2 } & \text { CASO 3 } \\ 1,000 & (1,000) & 1,000 \\ (1,000) & 500 & --\cdot \\ & & (2,000) \\ ----- & ----- & ----- \\ (--) & (\mathbf{5 0 0}) & (\mathbf{1 , 0 0 0})\end{array}$

$\begin{array}{ccc}(-.-) & (200) & (400) \\ -.- & -.- & 800 \\ ------ & ----- & ------ \\ & & \\ (-.-) & (\mathbf{2 0 0}) & \mathbf{4 0 0} \\ ==== & ==== & ====\end{array}$

$\begin{array}{ccc}1,000 & 1,000 & 1,000 \\ -.- & 200 & 400 \\ ---- & ----- & ------ \\ \mathbf{1 , 0 0 0} & \mathbf{8 0 0} & \mathbf{6 0 0} \\ ===== & ===== & ====\end{array}$

Considero razonable que dicha clasificación, de ser material, debería mostrarse en una partida separada de los Estados Financieros, con una nota a los mismos, divulgando la naturaleza y el efecto que originaron dichas partidas.

Respecto al estado de resultados, la gran mayoría de las Compañías muestra una línea que es denominada impuesto a la renta, y suelen diferenciarla con una nota en la cual describen la forma de cálculo y la situación que afecta la misma. No considero que sea apropiado mostrar en cuentas separadas en el estado de resultados los efectos impositivos de una diferencia temporaria, respecto de una pérdida tributaria; debido a que se hace complejo en los casos en que tenemos más de una partida temporal que tenga efectos impositivos, y que tendrá consideración contable en el futuro. Y aun cuando sea sólo una sola partida, considero más conveniente efectuarlo en una nota a los Estados Financieros, en la cual se muestren todos los efectos y la naturaleza de los mismos, así como los criterios de probabilidad, y forma de recuperación futura. 
Recordemos, que no sólo las diferencias temporarias podrían originar variaciones significativas. En la relación "gasto por impuesto a la renta vs. utilidad del ejercicio" se tendrá también, el efecto de las diferencias permanentes que pudieran existir, para las cuales, la exposición de su efecto, debe también expresarse en los Estados Financieros, por lo que se sugiere la siguiente exposición:

\begin{tabular}{|c|c|}
\hline Tasa corriente (efecto impositivo) & $40 \%$ \\
\hline Impacto de diferencias temporarias & * $(3 \%)$ \\
\hline Impacto de diferencias permanentes & $*(4 \%)$ \\
\hline Tasa real para el ejercicio & $33 \%$ \\
\hline & $======$ \\
\hline
\end{tabular}

Finalmente, cabe recalcar que bajo la premisa de que son efectos que revisten materialidad. Se deberá efectuar una adecuada exposición, de tal forma que permita una clara comprensión del hecho, para que tanto el Estado de Situación Financiera, como el Estado de Resultados, presenten la situación financiera y los resultados de las operaciones de acuerdo con las Normas Internacionales de Información Financiera, y sobre una base de uniformidad con los demás ejercicios que muestren estas particularidades.

\section{CONCLUSIONES}

1. El impuesto a la renta no es un costo de hacer negocios, por lo cual se hacen necesarios instrumentos como los que establece la NIC 12, para llevarlo a la categoría de una partida atribuible a todos los ingresos y gastos que participan en el resultado de operaciones.

2. Debemos reconocer la necesidad de que el impuesto a la renta, al ser un gasto atribuible a todas las partidas que participan en la generación de la utilidad o pérdida de gestión, represente un porcentaje razonable del resultado contable del ejercicio antes de impuestos, similar a la tasa tributaria que tenga el país en el cual se esté operando (en el Perú 30\%). Esto se logrará efectuando un manejo adecuado de las diferencias temporarias originadas por operaciones durante el ejercicio, que pudieran ser producto de decisiones gerenciales o de ausencia de las mismas, y que tengan impacto en la adecuada representación del resultado de operaciones.

3. Se debe difundir de manera adecuada las virtudes de esta Norma Internacional de Contabilidad $\mathrm{N}^{\circ} 12$, de manera simple como propugna este tratados, de tal forma, que desde los claustros universitarios, el potencial contador público, sea hábil en el manejo de los efectos impositivos que se derivan de la diferencia entre la base contable y la base fiscal, de un mismo activo o pasivo que genere una diferencia temporaria.

4. El tratamiento adecuado de los efectos impositivos de las diferencias temporarias desde el punto de vista del análisis de los rubros del estado de situación financiera, ayudará no solamente a mostrar un impuesto a la renta razonable en el año en el cual surja esta diferencia, sino ayudará definitivamente en el control de las reversiones de las que tengan impacto en el estado de situación financiera, y que sean llevadas de un periodo a otro.

5. La forma de hacer la distribución del impuesto a la renta entre periodos contables, se basa en el análisis de las diferencias temporarias y de las diferencias permanentes, entre la utilidad o pérdida contable y la utilidad o pérdida tributaria; y de la aplicación del posible efecto impositivo (el impuesto que correspondería pagar, o ahorrar por dicha diferencia) en los diversos períodos que afecta a los activos y pasivos en los estados financieros.

\section{REFERENCIAS BIBLIOGRÁFICAS}

1. ALEXANDER D., ARCHER S. (2006) Guía de NIC / NIIF. CCH. Incorporated. USA

2. CISS-WOLTERS Klewer S.A. (2013-2014) Todo Contabilidad. Grupo Wolters Editores. España.

3. ERNST \& YOUNG (2009) Principales diferencias U.S. GAAP-IFRS-NIF - Instituto Mexicano de Contadores Públicos. Mexico.

4. IFRS Foundation (2012) Normas Internacionales de Información Financiera IFRS Foundation Publication Department Unites Kingdon. 